\title{
Preliminary Study of LAMP Method for the Detection of Equine Infectious Anemia Virus
}

\author{
Jia-qi HAN ${ }^{1}$, Han ZHANG ${ }^{1}$, Qin TANG ${ }^{1}$, Ting-chuan LIU $^{1}$, Jing XU ${ }^{1}$, \\ $\mathrm{Li}$-rui $\mathrm{LI}^{2}$, Gang $\mathrm{YU}{ }^{1}$, Yin $\mathrm{WANG}^{1}$, Xue-ping $\mathrm{YAO}^{1}$ and Ze-xiao \\ YANG $^{1^{*}}$ \\ ${ }^{1}$ Sichuan Agricultural University, Chengdu, China \\ ${ }^{2}$ Chengdu Provincial Center for Animal Disease Prevention and Control \\ Chengdu, China \\ ${ }^{*}$ Corresponding author
}

Keywords: Equine infectious anemia, LAMP, Detection.

\begin{abstract}
To develop a rapid molecular biological method for the detection of equine infectious anemia virus (EIAV), a set of 4 primers were designed according to the conserved sequence fragment of the gag non-structure protein gene of EIAV published in GenBank, and the reverse transcriptase loop-mediated isothermal amplification (RT-LAMP) assay was established through identification of recombinant plasmid pMD-19T-gag which including target gene fragments, optimization of reaction conditions, sensitivity and specificity tests, and the validity test. The results showed the EIAV RT-LAMP detection method had a ladder-like pattern of amplification bands incubation at $63^{\circ} \mathrm{C}$ for 2 hours by using agarose gel electrophoresis, the sensitivity of the RT-LAMP could reach about 100 copies $/ \mu \mathrm{L}$ of target fragments, there was no amplification for Proteus, Actinobacillus, Salmonella, Escherichia coli, Stenotrophomonasmaltophilia and Streptococcus type II from equine detection by this approach. The RT-LAMP assay established here had good specificity and sensitivity, which is suitable for EIAV rapid detection.
\end{abstract}

\section{Introduction}

Equine infectious anemia(EIA), also called bog heat, is a fulminating infectious disease prevalent in equus animals caused by EIAV virus ${ }^{[1]}$. EIAV are subordinate to retroviruse division lentivirus category, the viral nucleic acid have normal chain with double strand RNA, can be divided into 2 parts. The viral nucleic acid of EIAV is RNA, but the virus proliferation is reliable on DNA .EIAV could cause horse, donkey and mule infection, and the infected animals are the source of this disease ${ }^{[2]}$. The symptom of the disease presents variety and with the course of disease changing greatly. In newly epidemic area acute type are common with high case fatality rate while in old epidemic area, the chronic type, recessive types dominate, and case fatality rate is low. Generally, there is no strict seasonal or regional features ,but mostly in aestivo-autumnal seasons and forest full of hematophagus and marshland as well, and it chiefly presents local prevalence or interspersed occurrences. EIAV was first found in France in 1843, having been transported around the world after two world wars. In 1931 EIA was brought into northeast and north of China from Japan, and in 1954 and 1958 this disease was incoming again for the import of horses from the Soviet Union. The prevalence of EIAV in history have surpassed half a century, EIAV was prevalent in 22 provinces in China and had caused enormous financial loss to agriculture and animal husbandry 
production. The disease has been listed as B type of infectious disease by OIE and China. EIAV was separated successfully in 1965 by veterinarian university of the Chinese People's Liberation Army and then developed two specific diagnostics, EIAV complement fixation test and agar diffusion test, and in 1975, Veterinary Research Institute of Haerbin academician initiated EIA leukocyte living vaccine ${ }^{[3]}$. Through immunization, monitoring, etc multiple comprehensive prevention and control measures the EIAV prevention and control work gained significant effect. In recent years, no clinical cases of EIAV were found nationwide, and the positive relevance ratio of EIAV is descending year by year. But the the surveillance and detection for disease is still especially important.

The commonly used method for diagnosis of EIA includes, comprehensive diagnosis, complement fixation test, agar-diffussion test, fluorescent antibody test and indirect ELISA test, etc ${ }^{[4,5]}$, and with the pace of molecular biological techniques such as PCR,RT-PCR and fluorescent PCR are also applied in the diagnosis and detection of disease. Although these methods are desired detection methods they also possess some limitations such as time-consuming and facility needing specific requirements. loop-mediated isothermal amplification( LAMP)is an new style isothermal nucleic acid amplification ,a new genetic diagnosis developed by Japanese scholar Notomi in 2000 and published on journal of Nucleic Acids Res ${ }^{[6,7]}$. LAMP techniques, by utilizing 4 different specific primers for the recognition of 6 specific location of target gene completing amplified reaction at isothermal condition. The amplification of gene and detection for products complete by one step ${ }^{[8,9]}$, with traits of high specificity, promote and sensitive, high efficient, and ease of operation, etc ${ }^{[10-14]}$. And then it received the worldwide concern ${ }^{[15,16]}$. As a novel nucleic acid amplification method, there is no reports on the LAMP methods for EIAV. In this study a reverse transcriptase loop-mediated isothermal amplification (RT-LAMP) assay was preliminary established and evaluated for EIAV diagnosis after a serial of tests including identification of target gene fragments, optimization of reaction conditions, sensitivity tests, specificity tests, and the validity test.

\section{Materials and Methods}

\section{Reagents and Samples}

dNTP Mixture,DL2000 marker were provided by the TaKaRa Biotechnology(Dalian) Co., Ltd. 2xTaq PCR Mastermix,DH5 $\alpha$ competence, TIAN premini Plasmid Kit(DP103), and TIANgel Midi Purification Kit(DP209)were provided by the Chengdu Feike Biotechnology (Tiantai) Co., Ltd. Bst 2.0 DNA polymerase (M0275) was provided by Beijing New England Biolabs Co., Ltd. Betaine(Z0041-1)was provided by Shanghai Kayon Biotechnology Co., Ltd. Equine infectious anemia test antigen (NECVB), Haerbin engineering research center of national animal disease biological products Co., Ltd. gag gene fragments of EIAV (pMD-19T-gag), constructed by Sichuan Agricultural University animal quarantine Lab. etc.

\section{Primer Design and Preparation}

According to the gag gene sequences of EIAV published in GenBank, 2 specific primers(P1/P2) for EIAV recombinant plasmid pMD-19T-gag PCR identification, and 4 LAMP primers for EIAV detection (listed in Table 1) were carefully designed using 
DNA Star and Primer Explorer V4 software (http://primerexplorer.jp/e/) subsequently. All of these primers were prepared by the TsingKe Co., Ltd

Table 1. Primers used for EIAV RT-LAMP study

\begin{tabular}{clcc}
\hline $\begin{array}{c}\text { Task and } \\
\text { Primer name }\end{array}$ & \multicolumn{1}{c}{ Sequence (5'-3') } & $\begin{array}{c}\text { Products } \\
\text { Size(bp) }\end{array}$ & $\begin{array}{c}\text { Reference } \\
\text { sequence }\end{array}$ \\
\hline EIAV PCR & & & \\
identification & & 364 & GQ923952 \\
F & TCAAGAAGTTAGAGAAGGTG & & \\
R & CTGTTTCCAGCCCCATCTA & & \\
EIAV RT-LAMP & & & \\
EIAF3 & CTTTGTWAAAGAAAARGACTGG & \\
EIAB3 & TGGATATTCYTCAKRGGGCTC & \\
EIAFiP & $\begin{array}{l}\text { ACCATGTTYTTTCAAAGGCYTCTARYTG } \\
\text { AGGGATGTCMTYCCA }\end{array}$ & \\
EIABiP & TGTRGKAGATGGAAARGCAWGCTYTTA & & \\
& GGAGCTGRWAWG & \\
\hline
\end{tabular}

\section{Recombinant Plasmid pMD-19T-gag Identification}

To identify the conserved target gag gene fragment of EIAV, the constructed recombinant plasmids pMD-19T-gag was identified by were subsequently identified by PCR (using F/R as primers) and by sequencing analysis as described in ${ }^{[17]}$. The PCR reaction volume $(50 \mu \mathrm{L})$ containing $25 \mu \mathrm{L}$ of $2 \times$ Taq PCR MasterMix, $1 \mu \mathrm{L}(10 \mu \mathrm{mol} / \mathrm{L})$ of each of the primers $(\mathrm{F} / \mathrm{R}), 1 \mu \mathrm{L}$ of recombinant plasmids and $22 \mu \mathrm{L} \mathrm{ddH}_{2} \mathrm{O}$. The PCR procedure program were: denaturing at $95^{\circ} \mathrm{C}$ for $5 \mathrm{~min}$, followed by 35 cycles at $94^{\circ} \mathrm{C}$ for $40 \mathrm{~s}, 52^{\circ} \mathrm{C}$ for $30 \mathrm{~s}$, and $72^{\circ} \mathrm{C}$ for $30 \mathrm{~s}$, then terminated by an elongation at $72^{\circ} \mathrm{C}$ for $10 \mathrm{~min}$. In all, $5 \mu \mathrm{L}$ of PCR products were analyzed using $10 \mathrm{~g} / \mathrm{L}$ agarose gel electrophoresis.

\section{Basic LAMP Reaction}

The RT-LAMP reaction mixture $(25 \mu \mathrm{L})$ contained Thermopol Buffer $(10 \times) 2.5 \mu \mathrm{L}$, Bst DNApolymerase $1 \mu \mathrm{L}, \quad$ betaine $\quad(8 \mathrm{mmol} / \mathrm{L}) 2.5 \mu \mathrm{L}, \quad \operatorname{dNTP}(2.5 \mathrm{mmol} / \mathrm{L}) \quad 8 \mu \mathrm{L}$, $\operatorname{Mgcl} 2(25 \mathrm{mmo} / \mathrm{L}) 3 \mu \mathrm{L}, \mathrm{F} 3 / \mathrm{B} 3(5 \mu \mathrm{mol} / \mathrm{L}) 1 \mu \mathrm{L}$, Fip/Bip $(25 \mu \mathrm{mol} / \mathrm{L}) 2 \mu \mathrm{L}, 1 \mu \mathrm{L}$ of positive recombinant plasmid pDM19-T-gag which was extracted according to the instructions of the plasmid extraction kit TIAN premini Plasmid Kit(DP103)as template, and add $\mathrm{ddH}_{2} \mathrm{O}$ to $25 \mu \mathrm{L} .65^{\circ} \mathrm{C} 2 \mathrm{~h}, 80^{\circ} \mathrm{C} 2 \mathrm{~min}$. The reaction mixture was incubated at $65^{\circ} \mathrm{C}$ for 2 $\mathrm{h}$, then heated at $80^{\circ} \mathrm{C}$ for $2 \mathrm{~min}$ in a laboratory water bath (DSY21-8, China), and the amplified products were analyzed in $15 \mathrm{~g} / \mathrm{L}$ agarose gel in TAE buffer. The results were also visualized directly with the naked eye according to the white precipitate of magnesium pyrophosphate generated in the reaction or the green color changed with $254 \mathrm{~nm}$ UV light by the addition of diluted SYBR Green I. To confirm the specificity of amplification products, $20 \mu \mathrm{L}$ of the reaction mixture which containing $3 \mu \mathrm{L}$ of the LAMP products was digested with HindIII and EcoR II at $37^{\circ} \mathrm{C}$ for $2 \mathrm{~h}$.

\section{Optimization of EIAV RT-LAMP Reaction Conditions}

In the optimum conditions assay, the RT-LAMP reaction mixture as described in above were used, and the reaction conditions were determined to be as follows: a gradient of incubation degree from $60^{\circ} \mathrm{C}$ to $65^{\circ} \mathrm{C}$ for $90 \mathrm{~min}$, and at the determined incubation degree for $150 \mathrm{~min}, 120 \mathrm{~min}, 90 \mathrm{~min}$ and $2 \mathrm{~h}$, respectively. In all, $5 \mu \mathrm{L}$ of RT-LAMP products were analyzed using $15 \mathrm{~g} / \mathrm{L}$ agarose gel electrophoresis. 


\section{LAMP Sensitivity Test}

Using the recombinant plasmids constructed above as standard control samples, The pMD-19T-gag, which concentration were determined by a ND-1000 ultraviolet spectrophotometer (Nano Drop Co., Ltd, USA),were diluted in a 10-fold serial, and each dilution was tested by the LAMP using the determined reaction conditions above. The number of target gene fragment molecule copies in each reaction was calculated according to, then the sensitivity of RT-LAMP was accessed ${ }^{[17]}$.

\section{LAMP Specificity Test}

The specificity tests of EIAV LAMP was carried out through comparing the pMD-19T-gag to Proteus bacillus, Actinobacillus, Salmonella, Escherichia coli, Streptococcus aeruginosa and Streptococcus type II etc common pathogens. RNA and DNA (cDNA) templates were prepared using a commonly reported method from ${ }^{[17]}$.

\section{Validity Test}

To assess the feasibility of the target gag gene fragment in pMD-19T-gag for RT-LAMP detection, the validity test was carried out using RNA extracted from Equine infectious anemia test antigen (NECVB)(EIAV) as template according to the reaction condition determined above.

\section{Result}

\section{Identification of EIAV Target Gag Gene Fragment}

The constructed plasmids pMD-19T-gag was identified by PCR (using F/R as primers) and sequencing analysis as described in the method section above, The results showed a DNA band about364 bp in the length (see Fig. 1, lane1) was amplified by PCR, and the sequencing results showed the target DNA fragment had a 100\% homology with the reference sequences(GQ923952). The result suggests that it is reliable to use the pMD-19T-gag including the EIAV gag target fragment.

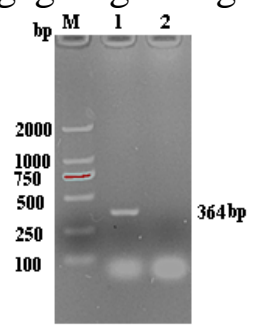

Figure 1. PCR identification of pMD-19T- gag

M: DNA marker DL2000; 1: pMD-19T-gag PCR products; 2: Negative control

\section{Basic RT-LAMP reaction}

The EIAV basic RT-LAMP was carried out using pMD-19T-gag as template and incubated at $65^{\circ} \mathrm{C}$ for $2 \mathrm{~h}$, the results are shown in Fig2. The agarose gel electrophoresis analysis of the LAMP reaction product showed that the DNA product of the ladder-like band was amplified (Figure 2A, lane 1) and the $\mathrm{ddH}_{2} \mathrm{O}$ negative control had no amplified bands (Figure 2A, lane 2) .The positive reaction tube (Fig. 2B, tube3) showed a significant turbidity compared to the negative reaction tube (Fig. 2B, tube4), and after the addition of SYBR Green I, the positive reaction tube showed fluorescence green (Fig. 2C, tube5) under ultraviolet light $(254 \mathrm{~nm})$, and the control reaction tube (Figure 
2C, tube6) no fluorescence produced. And the EIAV LAMP positive reaction product could be digested by restriction enzyme digestion with HindIII and EcoRI become the smaller digestion products (Fig.3, lane 2).

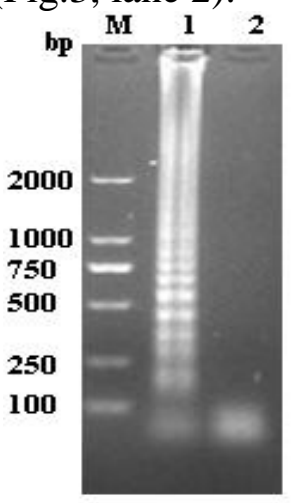

A

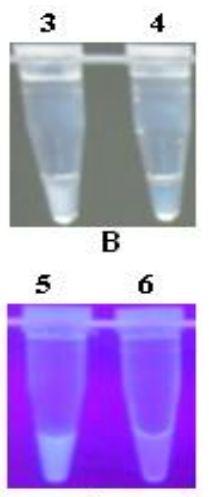

Figure 2. Results of EIAV basic LAMP

A:Agarose gel electrophoresis; B:Visual inspec- tion by turbidity; C:visual inspection by adding SYBR GreenI; M: DNA marker DL2000;1,3,5: RT-LAMP reaction products of pMD-19T-gag; 2,4,6: Negative control (ddH2O as template);

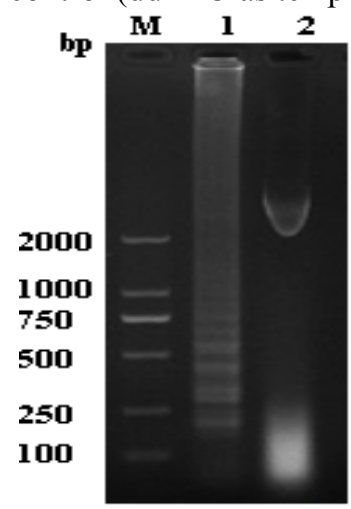

Figure 3. The digestion result of pMD19-T-gag LAMP products

M: DL2000 DNA Marker; 1: pMD19-T-gag LAMP products; 2: The digestion product of positive LAMP products digested with HindIII and EcoRI.

\section{Optimization of the RT-LAMP Reaction Conditions}

The temperature gradient screening test and the reaction time were optimized for the EIAV LAMP reaction according to the procedure section above, The results showed that the reaction temperature is similar to that of the reaction products at $62^{\circ} \mathrm{C}$ (Fig.4A, lane 3) and $63^{\circ} \mathrm{C}$ (Fig.4A, lane 4), better than the other temperatures (Fig.4A, lanes 1, 2, $5,6)$. There' re no significant difference between the $2 \mathrm{~h}$ (Fig.4B, lane 8) and 150min (Fig.4B, lane 9) amplification products, which are better than the 90min (Fig.4B, lane 7) products. Therefore, in order to ensure the specificity of the reaction and shorten the reaction time, the reaction conditions were determined as $63^{\circ} \mathrm{C} 2 \mathrm{~h}, 80^{\circ} \mathrm{C} 2 \mathrm{~min}$. And the $25 \mu \mathrm{L}$ reaction volume as described in the method section above was determined, and the optimum RT reaction volume components and conditions were the same as the instructions of Primerscript RT reagent Kit. 


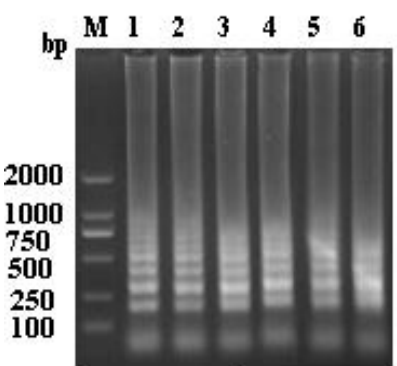

A

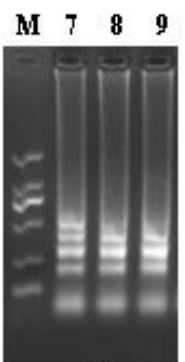

B

Figure 4.The optimization of LAMP reaction conditions for EIAV detection

A: The EIAV LAMP reaction temperature optimization result; B: The EIAV LAMP reaction time optimization result ;M:DL2000 DNA Marker; $1: 60^{\circ} \mathrm{C} 2 \mathrm{~h} ; 2: 61^{\circ} \mathrm{C} 2 \mathrm{~h} ; 3: 62^{\circ} \mathrm{C} 2 \mathrm{~h} ; 4: 63{ }^{\circ} \mathrm{C} 2 \mathrm{~h} ; 5: 64{ }^{\circ} \mathrm{C}$ $2 \mathrm{~h} ; 6: 65^{\circ} \mathrm{C} 2 \mathrm{~h} ; 7: 63^{\circ} \mathrm{C} 90 \mathrm{~min} ; 8: 63^{\circ} \mathrm{C} 120 \mathrm{~min} ; 9: 63^{\circ} \mathrm{C} 150 \mathrm{~min}$.

\section{Sensitivity and Specificity of EIAV LAMP}

In the sensitivity test, the pMD-19T-gag diluted at different concentrations were tested, The results(as shown in Fig.5) showed the ladder-like pattern could be observed clearly in lane 1 to lane7(100 copies/ $\mu \mathrm{L})$, indicating that the LAMP detection limit was 100 copies $/ \mu \mathrm{L}$ of the target gene fragments. The specificity test results (as shown in Fig.6)showed that only the EIAV recombinant plasmid pMD19-T-gag was positive for the detection(Fig.6, lane 1), and there were no amplification for Salmonella, Proteus, Aeromonas, Escherichia coli, Escherichia coli, Actinobacillus and Streptococcus type II detection(Fig.6, lane 2-7), which indicated the good specificity of EIAV LAMP.

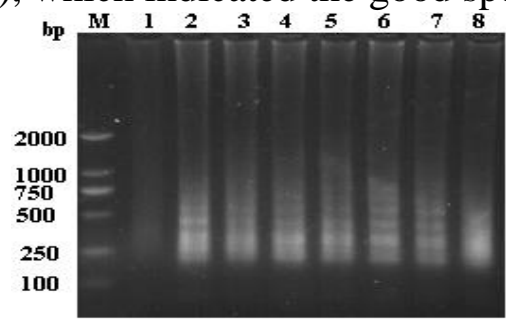

Figure 5.Sensitivity test of the EIAV LAMP

M:DL2000 DNA Marker; 1:Negative control; 2:Positive control; 3:1 $\times 10^{6}$ copies $/ \mu \mathrm{L} ; 4: 1 \times 10^{5}$ copies $/ \mu \mathrm{L} ; 6: 1 \times 10^{4}$ copies $/ \mu \mathrm{L} ; 6: 1 \times 10^{3}$ copies $/ \mu \mathrm{L} ; 7: 1 \times 10^{2}$ copies $/ \mu \mathrm{L} ; 8: 1 \times 10^{1}$ copies $/ \mu \mathrm{L}$

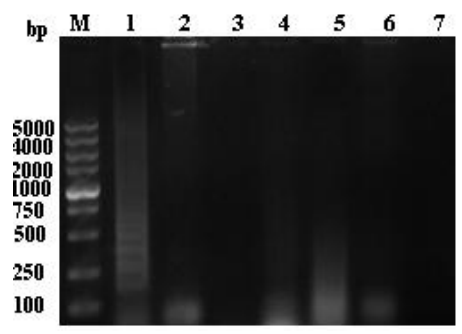

Figure 6. Specificity Test of the EIAV LAMP

M: DL5000 DNA Marker;1:pMD19-T-gag;2: Salmonella; 3: Proteus; 4: Aeromonas; 5: Escherichia coli; 6: Actinobacillus; 7: Streptococcus type II.

\section{Validity Test}

The validity test showed a positive result for RNA extracted from Equine infectious anemia test antigen $(\mathrm{NECVB} 尺)(E I A V)$ detection, which indicates the feasibility of this method for EIAV detection. 


\section{Discussion and Conclusions}

The new type of nucleic acid amplification LAMP, compared with the 2 conserved regions corresponding to the primer design for PCR. The primer design with LAMP is targeted at 6 conservative region of target gene, for 4 synthesis strips (including 2 strips of outer primer F3,B3, and 2 ring-shaped strips of inner primer Fip, Bip), consequently is stronger reaction specificity. And LAMP requires low for test apparatus and interpretation, only one isothermal equipment for amplification , and the result of amplification can be observed by naked eyes, and turbidity changes resulting from white magnesium pyrophosphate sediment can be interpreted directly, which made LAMP promising in application of molecular detection. LAMP reaction is conducted at constant temperature, not only convenient and quick, the requirements for apparatus is obviously lowered, and with widespread applicability.

Taking into account the risk of EIAV spread, In this study, based on the EIAV gag gene fragments artificially synthesized by overlapping-PCR. Then the synthetic gene fragments were used to optimize the reaction conditions, specificity and sensitivity tests. And use the horse infectious anemia agar diffusion test antigen virus isolate to access the LAMP detection validity, EIAV LAMP detection technology was established successfully. The results showed The LAMP method has good sensitivity and specificity, the reaction could be finished at $63^{\circ} \mathrm{C}$ within $2 \mathrm{~h}$. Its detection limits is up to 100 copies/ $\mu \mathrm{L}$, there is no specific amplification response for Proteus, Actinobacillus, Salmonella, Escherichia coli, Stenotrophomonasmaltophilia and Streptococcus II. To our knowledge, This is the first reported about the LAMP test of EIAV detection in China, and provides a scientific, reliable, rapid and specific, sensitive and sensitive detection method and technical reserve for EIAV detection.

\section{Acknowledgement}

This study was supported by Grants from the planning subject of the College students' innovative training program project of Sichuan agricultural university (1510626062), 'the twelfth five-year-plan' in national science and technology for the rural development in China (2013BAD12B04). Qin Tang, Han Zhang, Yin Wang, Xue-ping Yao and Li-rui Li should also be considered as first authors, Corresponding author: Ze-xiao Yang, Yin Wang.

\section{References}

[1] YM Zhu, HY Wang and W Guo, Development of an indirect ELISA for detecting the antibody of immunodominant region of transmembrane envelope protein of EIAV, J. Chinese Journal of Preventive Veterinary Medicine, 28 (2006) 191-196, in Chinese.

[2] TX Shen, Z Xu and YS He, Study on the immunity of equine infectious anemia, J. Chinese Journal of Agricultural Sciences, 4 (1979) 1-15, in Chinese.

[3] FY Chen, Veterinary infectious diseases, Fifth edit, Beijing, China Agriculture Press, 2008, in Chinese.

[4] S Ha, CM Dai and L Wang, The application of EIAV ELISA (indirect method) detection method for horse propagation, J. Chinese Veterinary Science Technology, 22 (1992) 21-22. 
[5] YK Dai, BS Zhang and S Ha, The specificity test in EIAV AGP negative ELISA positive disease horse serum, J. Chinese Veterinary Science Technology, 24 (1994) 3-4.

[6] T Notomi, H Okayama, H Masubuchi, T Yonekawa, K Watanabe, Loop-mediated isothermal amplification of DNA, J. Nucleic Acids Research, 28 (2000) 63.

[7] T Cheng, X Liu and X Chang, Establishment and application of loop-mediated isothermal amplification for detection of Aeromonashydrophila, J. Veterinary Science in China, 37 (2007) 1013-1016.

[8] T Xin, SH Hou, H Jia, XY Guo and JB Ding, Development of a novel RT-LAMP assay for porcine reproductive and respiratory syndrome virus, J. Scientia Agricultura Sinica, 43 (2010) 185-191.

[9] QM Li, XJ Ma, HC Gao, R Zhou and ZZ Kuang, Evaluation of reverse transcription loop-mediated isothermal amplification for detection of avian influenza A H5N1 virus, J. Chinese Journal Of Virology, 24 (2008) 178-184.

[10] Q Xu, X Sun, Y Zhao and Y Pan, Development of Loop-Mediated Isothermal Amplification (LAMP) Method for Detection of Vibrio parahaemolyticus, J. China Biotechnology, 27 (2007) 66-72.

[11] ZF Qin, SL Zeng, R Zhou-Xi, B Cheng and CF Cao, Development of a novel RT-LAMP assay for foot-and-mouth disease virus, J. Chinese Journal of Preventive Veterinary Medicine, 30 (2008) 375-378.

[12] SM Zhu, WU Jia-jia, XU Chi, QU Jiong and W Cheng, Rapid Detection of Salmonella spp. by Loop-Mediated Isothermal Amplification Method, J. Modern Food Science and Technology, 24 (2008) 725-730.

[13] YW Zhang, XN Li, PP Guo, P Fu and JH Li, Fluorescent reagent in detection of porcine reproductive and respiratory syndrome virus by loop-mediated isothermal amplification (LAMP) , J. Journal of Agricultural Biotechnology, 18 (2010) 508-513.

[14] W Li, G Li, XJ Fan, K Zhang and FQ Jia, Establishment of a rapid method for detection of peste des petits ruminants virus by a reverse transcription loop-mediated isothermal amplification, J. Chinese Journal of Preventive Veterinary Medicine, 31 (2009) 374-378.

[15] Z Yang, H Zeng, Y Wang, X Yao and K Wang, Establishment of a RT-LAMP Method for the Rabbit Hemorrhagic Disease Virus Detection, Biotechnology, J. An Indian Journal, 8 (2013) 164-269.

[16] Z Yang, Y Hou, H Zeng, X Yao, Y Wang, H Liu, B Liu, X Wu and B Peng. Development and Application of LAMP Method for Porcine Circovirus type2 Detection, J. Chinese Veterinary Science,44 (2014) 152-158.

[17] Z Yang, Z Meng, H Zhang, Xiang, Y,Liu Y Luo, X Yao, Y Wang, L Zhou and Y Li, Preliminary Study on the RT-LAMP assay for Rabbit Hemorrhagic Disease Virus type 2 Detection,J. Advances in Biological Sciences Research, 3 (2017) 127-132. 